\title{
Bonheur et bienveillance : l'école française en quête de félicité
}

Caractérisation des pratiques qualifiées de bienveillantes par des membres du personnel enseignant de collège

Happiness and benevolence: the French school in search of happiness.

Characterizing practices according to a sample of interviews with some

Secondary schools members of staff

\section{Gwénola Réto}

\section{(2) OpenEdition}

Journals

Édition électronique

URL : https://journals.openedition.org/trema/5361

DOI : 10.4000/trema.5361

ISSN : 2107-0997

Éditeur

Faculté d'Éducation de l'université de Montpellier

Référence électronique

Gwénola Réto, «Bonheur et bienveillance : l'école française en quête de félicité », Tréma [En ligne], 52 |

2019, mis en ligne le 01 septembre 2019, consulté le 16 janvier 2023. URL : http://

journals.openedition.org/trema/5361 ; DOI : https://doi.org/10.4000/trema.5361

Ce document a été généré automatiquement le 16 janvier 2023.

Tous droits réservés 


\title{
Bonheur et bienveillance : l'école française en quête de félicité
}

\author{
Caractérisation des pratiques qualifiées de bienveillantes par des \\ membres du personnel enseignant de collège \\ Happiness and benevolence: the French school in search of happiness. \\ Characterizing practices according to a sample of interviews with some \\ Secondary schools members of staff
}

\section{Gwénola Réto}

\section{Introduction}

1 La place donnée au bonheur dans notre société interroge, jusqu'à pousser certains chercheurs à dénoncer l'essor d'une happycratie inquiétante (Cabanas, Illous, 2018). La quête du bonheur, qui remporte un vif succès dans le champ du développement personnel, est accompagnée dans son sillage par la préoccupation du bien-être et par un engouement pour une autre notion, la bienveillance. Derrière cette bonne fortune sémantique se pose la question de l'eudaimonia, de « la réussite de la vie individuelle, que chacun veut, mais sans vraiment savoir, le plus souvent, en quoi elle consiste" (Spaemann, 1997). Au-delà de la démarche individuelle, se pose également la question du bien vivre ensemble et de la quête d'une félicité qui gagne jusqu'au rang de l'École.

2 Partant de la mise en relief de l'émergence de cette notion dans la société et dans le cadre de l'École, nous contextualiserons la réflexion en montrant l'appel fait à la bienveillance qui existe au sein du système scolaire, notamment dans le projet pour la refondation de l'École française. Nous interrogerons le sens de cet appel puis, confrontés au constat de manque de conceptualisation de la bienveillance, nous en proposerons une clarification en prenant appui sur le champ de la philosophie, et plus précisément de l'éthique. La bienveillance interroge tant le système que les pratiques. Mais comment opérationnaliser la notion dans les pratiques professionnelles ? S'agit-il d'un appel à de nouvelles modalités relationnelles? La bienveillance n'est-elle qu'une 
histoire de qualité personnelle ou bien s'inscrit-elle dans des modalités pédagogiques ou didactiques spécifiques? Quel rapport entretient-elle avec l'autorité et l'exigence? Quelle posture et quelles compétences professionnelles induit-elle? Afin de caractériser les pratiques relevant de la bienveillance à l'École, nous prendrons alors appui sur une recherche qualitative $e^{1}$ menée auprès de membres du personnel enseignant travaillant dans des collèges. Nous mettrons en évidence différentes formes de bienveillance identifiables dans le discours sur les pratiques professionnelles de ces membres, et les incidences sur la posture et le développement de ces derniers. Enfin, en conclusion, nous discuterons ces résultats et interrogerons les changements liés à cet appel à la bienveillance et aux notions qui lui sont reliées pour le système scolaire.

\section{L'inflation de la notion de bienveillance, du champ social au champ Éducatif}

3 L'inflation du terme «bienveillance " invite à s'interroger. Dans un premier temps, nous montrerons l'émergence de la notion de bienveillance dans la société, puis dans le champ de l'éducation et dans le cadre de l'École. Nous situerons cet essor en lien avec la quête contemporaine de bien-être et interrogerons ce dont cet appel à la bienveillance est le symptôme tant dans le champ social que dans celui plus spécifique de l'École.

\section{La bienveillance, un terme " tendance »}

4 La notion de bienveillance est à la mode et connait un essor particulier dans le domaine éditorial, notamment dans les ouvrages appartenant à la catégorie du développement professionnel. Plus de 60 livres mentionnant le terme ont été publiés ces 10 dernières années, alors que le siècle précédent se caractérisait par l'absence de recours à ce terme. L'appui sur les travaux menés en sociologie et en psychologie visant à caractériser la société contemporaine montre que cet essor peut s'expliquer par différents facteurs tels que la quête effrénée de bien-être (Marquis, 2016), la surabondance du sens (Ehrenberg, 1998), la mutation du rapport au temps (Aubert, 2004) ou encore une certaine altération des relations sociales (Poché, 2015). Touché dans son identité profonde, l'individu contemporain est sommé de travailler sur luimême. Fragmenté et dérouté, la bienveillance peut lui apparaitre comme une force restauratrice adaptée, lui offrant une voie plus altruiste et l'ouvrant à une quête d'authenticité vers un horizon moral qui l'oriente (Taylor, 1994). Cependant, la notion est peu stabilisée, permettant alors un champ d'interprétation large et une flexibilité qui contribuent à son succès mais qui interrogent sur sa signification plus profonde.

\section{L'émergence de la bienveillance en éducation}

5 L'émergence de la notion de bienveillance se manifeste particulièrement dans les différents espaces éducatifs. Son succès s'atteste dans les champs professionnels de l'humain, en lien avec la bientraitance (Moulias et al., 2010), et cette notion est insérée dans les politiques éducatives urbaines. Elle connaît également un engouement dans le champ de l'éducation familiale et cette émergence peut être reliée aux interrogations sur la parentalité de la part d'individus en quête de repères quant à leurs compétences éducatives. 
6 S'agissant du cadre scolaire, la notion se révèle centrale dans la réflexion pour la refondation de l'École française. L'appui sur une recension et une analyse de ses occurrences dans les différents textes institutionnels publiés par le ministère de l'Éducation nationale de 2013 à 2015 permet de montrer que la bienveillance est devenue un attendu pour l'institution dans son ensemble, en lien avec les préoccupations du climat scolaire et du bien-être à l'École (Réto, 2016 et 2017). La bienveillance constitue également un attendu relationnel structurant, à relier à la quête d'une nouvelle forme d'autorité, et elle représente un nouvel attendu pour le personnel enseignant. En effet, le terme a fait son apparition dans le dernier référentiel définissant ses compétences, paru en juillet 2013. Alors que le précédent référentiel avait recours au terme autorité, ce dernier attend des enseignants qu'ils «installe[nt] avec les élèves une relation de confiance et de bienveillance » (MEN, 2013).

Cependant, son absence de caractérisation conduit à interroger son émergence au regard des évolutions et des mutations que rencontre l'École. La notion apparaît en effet à un moment où règne un malaise institutionnel important, où l'École se trouve elle aussi « sous pression » et confrontée à ses carences, ce qui explique la réclamation de changements. Les constats de l'échec du projet égalitaire de l'École (Mons, 2016) et d'un climat scolaire dégradé (Debarbieux, 2015) rejoignent ceux faits depuis une trentaine d'années. Ils font écho, entre autres, à la dénonciation de la violence symbolique et invisible (Delfrance, 1992) et des pratiques d'humiliation qui laissent à penser que l'École serait un espace de non-droit (Merle, 2005). L'appel à la bienveillance s'inscrit aussi dans un vaste projet qui vise à réaffirmer l'importance de la formation aux valeurs à l'École et la part axiologique de l'éducation (Bergounioux, Loeffel et Schwartz, 2013). Elle invite à de nouvelles formes d'apprentissages et de relations. Jellab (2018) souligne d'ailleurs que « la notion de bienveillance parait aujourd'hui aller de pair avec l'émergence de plusieurs catégories discursives mettant en jeu une sorte de care éducatif, comme les termes de bien-être, de climat scolaire apaisé, etc. » (p. 23).

8 L'appel à la bienveillance s'explique également par les avancées des recherches en neurosciences qui font le lien entre climat scolaire bienveillant et développement des apprentissages. Ainsi, Gueguen (2014) met en évidence le fait que la bienveillance facilite le travail de la mémoire et des apprentissages. Elle pointe aussi l'importance de la dopamine et de l'ocytocine sur la motivation et les apprentissages, en lien avec l'attention bienveillante qui relèverait alors d'une sorte de cercle vertueux. De même, les travaux de Rascle, Bergugnat, Florin et Guimard (2016) montrent que la qualité de vie des enseignants est dépendante de leurs interactions avec les élèves. Développer une relation bienveillante semblerait alors à même d'apporter des pistes très fécondes à la qualité relationnelle, elle-même nécessaire aux apprentissages.

9 Cependant, là encore, bien que pierre angulaire de la refondation, la bienveillance reste une notion peu définie et peu opératoire, laissant la voie à de multiples interprétations et ne donnant pas de prise à un possible déploiement dans la sphère éducative, malgré l'appel institutionnel et relationnel. 


\section{La bienveillance, une notion difficilement saisissable, pluridimensionnelle}

10 Pour pallier cette carence, nous faisons le choix de caractériser la bienveillance à partir du cadre de l'éthique, fondant cette option sur la constitution étymologique du mot (benevolens) qui met en tension deux notions fondamentales, le vouloir et le bien, ainsi que sur la place donnée à la bienveillance dans les écrits en philosophie morale. La bienveillance tient, en éthique professionnelle, une place particulière. D'ailleurs, pour Marsollier (2018), elle est « avec la tempérance, la justice, la confiance et le tact, l'une des vertus fondatrices de l'éthique éducative et pédagogique» (p. 64). Nous interrogerons dans un premier temps ses manifestations puis nous proposerons une caractérisation de la notion autour de quatre dimensions interreliées.

\section{Les manifestations de la bienveillance}

11 La bienveillance est définie comme « volonté réelle et efficace » (Foulquié et Saint-Jean, 1969, p. 72) et seule son effectuation peut permettre de révéler si l'efficacité visée a été atteinte. Toutefois, il n'existe pas de forme verbale à la bienveillance, ce qui contribue à rendre complexes sa traduction en actions et son analyse sur le terrain de la pratique. Elle n'existe en effet que dans son incarnation, concrétisée dans une attitude ou dans la réalisation d'un acte, permettant le passage de l'intention à la potentialité de sa réalisation. Elle demande à être actualisée dans les situations où se conjuguent ses deux formes: celle du «PAR bienveillance», qui prend appui sur son orientation éthique et celle du "AVEC bienveillance », centré sur l'agir et la pratique, permettant le passage à la manifestation (Massé, 1986). Si l'on se penche sur les cooccurrences du terme, on constate que la présence de la bienveillance s'atteste au travers d'une marque, d'une preuve, d'un témoignage, d'une attitude ou d'un signe qui se concrétise par une expression, la plupart du temps reliée au visage, qu'il s'agisse d'un air, d'un sourire ou d'un regard. Elle existe aussi dans des gestes. Elle peut également être associée à une attention, un examen, un traitement, une considération ou un accueil qui se caractérise par l'ouverture à l'autre. Dans le cadre de sa manifestation dans le langage, elle s'exprime dans l'émission, par le ton ou plus largement une parole bienveillante, comme dans la réception, par une réceptivité particulière. Ces cooccurrences montrent que la bienveillance se manifeste par une forme de qualité du comportement, par des manifestations très fines de la corporéité, dans une transcription de l'intériorité qui peut sembler ténue car sa traduction ne se fait jamais de manière spectaculaire.

12 La bienveillance semble davantage être perceptible dans la réception de son actualisation par un autre que par son propre auteur. Par cette reconnaissance d'une attitude perçue, reçue, interprétée et qualifiée comme telle par un autre s'opère le passage à la manifestation de l'intention à la bienveillance effective. Pour que la bienveillance de l'auteur puisse se manifester, elle demande ainsi à se concrétiser dans une attitude adressée et orientée vers le bien d'autrui, en réponse à un besoin et en prenant en compte son intérêt, en associant sa volonté à celle d'un autre et en l'exprimant par des actes, par des gestes et une posture sous-tendus par un sens éthique, permettant la conjugaison du PAR et du AVEC et nécessitant une addition de signes qui se constituent en faisceau à même d'être traduits dans une globalité qui peut 
alors être considérée comme étant de la bienveillance. Elle peut se manifester dans tout type de situation dès lors que cette dernière touche à la relation envers un autre ou plusieurs autres, au cœur des sphères tant privées que professionnelles.

Toutefois, les situations dans lesquelles la bienveillance se manifeste et se pratique ne permettent pas, selon cette entrée, de la penser en termes de gestes techniques, déclinables, mais plutôt en termes de manifestations dans une pratique relationnelle fondée sur une attitude et reliée à une conception d'autrui et de la responsabilité que l'on a par rapport à lui.

\section{Les quatre dimensions de la bienveillance}

Pour spécifier la bienveillance dans la relation éducative au sein du système scolaire, il apparait alors pertinent de prendre appui sur les apports des écrits en éthique professionnelle appliquée à l'enseignement en conciliant le déontologisme tempéré (Prairat, 2014) et le caring (Noddings, 1884). Il est alors possible de distinguer différentes dimensions complémentaires et interreliées pour caractériser la bienveillance. Nous en avons identifié quatre :

15 1) la dimension intentionnelle, d'abord. Elle s'appuie sur la volonté du bien tout en interrogeant l'intention dans sa tension avec l'action. Cependant, cette intentionnalité invite à l'inscrire dans des relations situées et finalisées au nom d'une responsabilité particulière. En effet, être bienveillant envers autrui, vouloir le bien d'autrui et le traduire en acte se fait dans toute situation où une personne identifie l'existence d'un besoin pour lequel elle estime qu'elle est en capacité d'apporter une réponse, dans un contexte donné, et au nom de quelque chose. Il s'agit tout à la fois de répondre $\mathrm{A}$ et de répondre $\mathrm{DE}$ au nom de sa responsabilité, ce qui implique de pouvoir et de devoir répondre de ses actes. Or on ne répond $\mathrm{DE}$ que de ceux avec qui nous sommes liés. Ce peut être un lien institué par la situation, par exemple un professionnel mandaté pour agir et qui intègre la bienveillance dans ses actes, en lien avec le cadre de sa profession, ou encore un parent qui agit au nom de la responsabilité éducative qui lui incombe. Ce peut être aussi plus largement au nom de sa conception de l'être humain, en lien avec un système de valeur personnel et une anthropologie qui fonde l'injonction à se sentir concerné par l'autre, qui appelle à exercer sa responsabilité en lien avec lui, qu'il s'agisse de l'autre proche ou de l'autre lointain.

En éducation, il s'agit d'une responsabilité et d'une exigence spécifique du fait des finalités éducatives inscrites dans le travail des enseignants. Il s'agit, pour le personnel enseignant, d'assumer sa responsabilité qui suppose " un "devant qui", un destinataire, quelqu'un qui puisse demander des comptes et soit accessible à des raisons " (Spaemann, 1997, p. 252). Il s'agit, pour la personne en responsabilité, de se préoccuper du « en vue de » et du « afin de » de l'autre, de veiller à son bien, à son bien-être et à son bien croître.

2) la dimension interactionnelle, ensuite, est fondée sur la connaissance de soi et la reconnaissance de l'autre, et elle questionne la possibilité de la réciprocité de la bienveillance. Dans la perspective éducative, elle doit être redéfinie puisque se pose de manière plus forte la question de la dissymétrie et qu'il s'agit alors de repenser une nouvelle forme de relation d'autorité entre l'enseignant et ses élèves. La relation bienveillante nécessite aussi d'être capable d'adopter une juste distance, différente selon les âges mais aussi selon chaque membre du groupe classe. 
17 3) la dimension affective est nourrie par la proximité de la bienveillance avec les sentiments. Elle interroge la tension entre la distance et la fusion et limite le risque de la confusion. Dans le champ éducatif, elle conduit à préciser la place des affects dans la relation éducative, puisque la personne qui enseigne doit se mêler des émotions, très importantes pour l'apprentissage, sans s'emmêler dans l'enchevêtrement émotionnel. Dans la relation avec les élèves, l'enseignant va mobiliser sa capacité à percevoir, évaluer et prendre en compte l'expression de l'affectivité des jeunes avec qui il travaille, mais aussi sa propre affectivité.

18 4) la dimension attentionnelle, enfin, articule la disponibilité et la réflexivité dans une attitude de veille et d'hospitalité. En éducation, être bienveillant nécessite une forte disponibilité car l'enseignant doit porter une attention spécifique dans le contexte particulier de la classe : une attention à la fois à chacun et à tous, ce qui exige de multiplier sa capacité d'attention. C'est d'ailleurs la condition nécessaire pour parvenir à identifier les besoins des élèves et à proposer une réponse ajustée à la situation. Cela nécessite également de laisser une place à l'intuition dans la prise en compte des situations éducatives.

19 Cependant, malgré cette clarification du concept de bienveillance en éducation, des éléments apparaissent comme restant à éclairer au niveau de la mise en œuvre concrète de la bienveillance dans les pratiques professionnelles du personnel enseignant.

\section{La bienveillance dans les pratiques : une « saisie » problématique}

Compte tenu des enjeux dont la notion se trouve investie, il nous a paru important de caractériser avec davantage de précision les pratiques des enseignants. Comme la bienveillance s'enracine pour partie dans l'intériorité, elle est de fait, peu aisée à "saisir ». Comment alors parvenir à enserrer plus finement ses manifestations dans la trame d'une recherche? Comment extraire de la pratique professionnelle des enseignants ce qui pourrait permettre de mieux comprendre son émergence dans leurs pratiques?

21 Pour répondre à ces interrogations, nous avons mené une recherche qualitative à visée compréhensive. Nous avons visé non la généralisation au sens statistique mais une contribution à la compréhension de la bienveillance dans sa spécificité scolaire. Compte tenu du peu de travaux menés à ce jour sur la bienveillance en éducation en contexte français, cette recherche a été exploratoire. Cette recherche a pris appui sur des entrevues afin de pouvoir prendre en compte les réalités à partir des interprétations que les professionnels construisent (Crahay, 2006). Notre choix s'est arrêté au terrain du collège ${ }^{2}$. Du fait de notre approche qualitative et de la visée compréhensive de la recherche, le recrutement des participants a été intentionnel, en fonction des buts poursuivis. Les personnes ont été recrutées « en fonction de leur expertise, pertinente par rapport à l'objet d'étude et parce qu'elles sont capables et intéressées à verbalisées celles-ci » (Savoie-Zajc, 2016, p. 349). Nous avons recruté des profils de participantes et de participants variés ${ }^{3}$, avec des responsabilités diverses et une implication de nature différente sur le terrain, sans viser de représentativité statistique (Miles et Huberman, 2003) mais en cherchant à ouvrir à la plus grande variété de pratiques. Il s'agissait d'entendre la manière dont des membres du personnel enseignant définissent la bienveillance en éducation et ce qu'ils disent mettre en œuvre. Nous avons ainsi visé à 
affiner la caractérisation du concept par la confrontation à leurs conceptions et à leurs représentations de ce qu'est la bienveillance en éducation, et ce qu'ils disent de leurs pratiques bienveillantes. Notre échantillon a donc été un échantillon de convenance. Comme l'objectif de notre recherche était de collecter les avis, les pratiques et expériences des professionnels, notre choix s'est porté sur l'entrevue de groupe plus propice à l'obtention de données pertinentes car nourries des controverses professionnelles qui peuvent naître des échanges (Geoffrion, 2016), ce que nous visions ici. Nous avons mené quatre entrevues semi-dirigées de groupe d'une durée d'une heure à une heure trente, avec des enseignants exerçant dans des collèges des Pays de la Loire. Nous présentons dans le tableau ci-après la composition des groupes ${ }^{4}$.

\begin{tabular}{|c|c|c|}
\hline \multicolumn{3}{|c|}{ Tableau : Composition des groupes d'enseignants pour les entrevues } \\
\hline 1 & Groupe 1 - Personnels du Collège A & $\begin{array}{l}\text { - Diane, } 50 \text { ans, professeure de Lettres } \\
\text { - Étienne, } 52 \text { ans, professeur de Mathématiques } \\
\text { - Lucas, } 38 \text { ans, professeur d'E.P.S. }\end{array}$ \\
\hline 2 & Groupe 2- Personnels du Collège B & $\begin{array}{l}\text { - Augustine, } 41 \text { ans, professeure de Mathématiques } \\
\text { - Fabrice, } 39 \text { ans, professeur de Technologie } \\
\text { - Marina, } 39 \text { ans, professeure de Mathématiques } \\
\text { - Nathalie, } 54 \text { ans, professeure d'E.P.S. } \\
\text { - Raphaël, } 50 \text { ans, professeur d'Espagnol }\end{array}$ \\
\hline 3 & Groupe 3 - Personnels du Collège $\mathrm{C}$ & $\begin{array}{l}\text { - Boris, } 31 \text { ans, professeur de Mathématiques } \\
\text {-Émilie, } 39 \text { ans, professeure d'Anglais } \\
\text { - François, } 43 \text { ans, professeur de Lettres } \\
\text { - Géraldine, } 40 \text { ans, professeure d'Histoire-Géographie } \\
\text { - Grégoire, } 44 \text { ans, professeur d'E.P.S. } \\
\text { - Richard, } 52 \text { ans, professeur de Technologie } \\
\text { - Thibaut, } 43 \text { ans, professeur d'E.P.S. }\end{array}$ \\
\hline 4 & Groupe 4 - Personnels du Collège D & $\begin{array}{l}\text { - Alice, } 47 \text { ans, professeure de Mathématiques } \\
\text { - Marie, } 55 \text { ans, professeure de Mathématiques } \\
\text { - Matthieu, } 45 \text { ans, C.P.E. } \\
\text { - Paul, } 31 \text { ans, professeur des écoles en S.E.G.P.A. } \\
\text { - Pascal, } 53 \text { ans, professeur de Technologie }\end{array}$ \\
\hline
\end{tabular}

Un guide d'entrevue a été construit selon la technique de Paillé (1991). Il comportait 4 $a^{2} s^{5}$, chacun décliné ensuite en différentes interrogations. Nous nous appuierons ici sur les deux premiers axes: la définition de la bienveillance et l'actualisation de la bienveillance dans les pratiques de classe. Les données ont donné lieu à une captation audio et ont été intégralement retranscrites. L'analyse qualitative des données s'est déroulée en deux volets successifs ${ }^{6}$. Ce processus d'analyse a permis de dégager des éléments de caractérisation de pratiques dites de bienveillance et déclarées mises en œuvre par le personnel enseignant à partir du discours des participantes et des participants, ce que nous allons désormais nous attacher à présenter. 


\section{Caractérisation des pratiques de bienveillance du personnel enseignant à partir de leurs discours}

L'examen attentif a montré que l'appel à la bienveillance est globalement bien accueilli. Les personnes interrogées n'ont pas toutes exprimé la même conception de la bienveillance ni témoigné des mêmes pratiques, et cela a constitué une richesse. Toutefois, nous faisons le choix de ne présenter ici que ce qui s'est dégagé de manière forte et convergente. Il est ainsi rapidement apparu que les personnes interrogées faisaient usage du mot bienveillance en lui attribuant différentes significations. Au fil de l'analyse des différentes entrevues, il a été possible d'identifier trois formes différentes dans les mentions de la bienveillance, chacune pouvant être caractérisée spécifiquement même si elles sont interreliées dans les pratiques. Nous les présenterons avant d'exposer un autre élément qui s'est dégagé: la dynamique d'ajustement qui préside aux pratiques déclarées par les enseignants.

\section{Les formes de bienveillance dans les pratiques selon les déclarations d'enseignants de collège}

L'analyse a permis de mettre à jour différentes formes de la bienveillance dont l'une est plus personnelle, les deux autres relevant de la professionnalité du personnel enseignant.

\section{La bienveillance comme qualité personnelle}

L'ancrage de la bienveillance dans une dimension personnelle apparait dans toutes les entrevues effectuées. Nous avons pu, de ce fait, identifier une forme de bienveillance envisagée comme qualité personnelle, c'est-à-dire reliée au caractère et la personnalité de chacun. Pour les personnes interrogées, cette forme de bienveillance renvoie en effet à une qualité "innée " selon Boris. Elle s'appuie notamment sur les valeurs personnelles, le goût des relations, le souci des autres, etc. Les personnes ont évoqué l'ancrage possible de cette forme dans leur biographie ainsi que la conjonction qui existe entre le caractère et le choix du métier. Ainsi, pour François, la bienveillance est «à la base des qualités à avoir » pour enseigner et le fait de posséder cette qualité orienterait la personne vers des métiers très relationnels.

Mais c'est insuffisant pour l'exercice professionnel. Lorsqu'il s'est agi de décliner les pratiques concrètes mises en œuvre dans le contexte scolaire, les enseignants ont illustré des formes beaucoup plus élaborées de bienveillance.

\section{La bienveillance comme art professoral}

A partir de l'analyse des données discursives, nous avons identifié une forme très construite et centrée sur le souci du développement des apprentissages des élèves que nous avons, de ce fait, appelée « art professoral ». Elle regroupe l'ensemble des moyens et procédés mis en œuvre par l'enseignant afin que les élèves développent les apprentissages visés. Diane souligne ainsi sa conscience de "transmettre aux élèves pour leur bien ». Les pratiques de différenciation et d'évaluation ont été largement documentées: il s'agit, pour le personnel enseignant, d'évaluer avec pertinence et 
précision les besoins des élèves afin de leur apporter l'aide et l'étayage nécessaires à leur développement. La gamme de pratiques décrite par les enseignants est étendue, et porteuse d'une forte exigence pour les élèves, mais aussi pour eux-mêmes, comme l'indique François :

Ce qui (...) est très difficile, c'est que pour comprendre l'élève, pour l'accompagner, pour savoir d'où il part, pour être effectivement bienveillant car ils ont tous des problématiques différentes, on rentre dans l'individualisation. [...]. Cela fait du coup autant de plans de vol différents qu'on a d'élèves.

Est également apparue, pour les personnes interrogées, l'importance de créer un climat relationnel propice aux apprentissages. Elles ont décrit, dans ce cadre, leur manière d'accueillir les élèves en classe, comme Lucas qui indique comment, dès l'entrée, sa façon de procéder veut signifier à l'élève "je te prends comme tu es ", ou les rites et routines mis en place avec les élèves pour leur apporter un cadre sécurisant avec « un relationnel ritualisé ", pour Émilie. Cela passe aussi par leur manière de manifester leur confiance aux élèves, de les accompagner « le plus justement possible " (Marina), et de les valoriser. Raphaël, évoquant les pratiques sous-jacentes au Cadre Européen des Langues, indique une évolution dans ses pratiques : « on bonifie tout ce qui est bien ». La présence de la félicitation a d'ailleurs une place importante dans les données. Son emploi peut être assimilé tout à la fois à un souci et un art de rendre heureux, permettant de renforcer le bonheur des élèves, mais aussi des personnels.

\section{La bienveillance comme incarnation du cadre éducatif}

La troisième forme identifiée est apparue souvent plus tardivement dans les entrevues, avec l'expression de la préoccupation d'une évacuation de l'autorité. Pourtant, les enseignants ont tous exprimé l'importance, dans leurs pratiques bienveillantes, du cadre et des limites à poser pour permettre un développement individuel et collectif. Partant de cette crainte, les personnes ont illustré la manière dont elles veillaient à développer une modalité relationnelle spécifique, refusant le rapport de force et visant l'instauration d'un cadre protecteur. Ainsi, pour Matthieu,

La bienveillance serait un cadre qui est capable de se déformer mais pas de se casser. [...]. C'est sécuritaire d'être dans un cadre. Sauf que les ados, par définition, ils n'aiment pas les angles. Et quand ça se déforme, c'est un peu plus simple, ça offre une souplesse sans qu'il y ait de sortie du cadre possible.

L'analyse des données a permis de mettre en évidence que les personnes interrogées s'appuient sur un «art du recadrage » spécifique, qui recouvre aussi les pratiques de sanction. Ces caractéristiques nous ont amenée à mettre en évidence l'expression d'une veillance particulière, qui se matérialise dans l'attention au développement des élèves et à leur devenir et dans un cadre finalisé par l'apprentissage de la vie collective, préparant au futur. Cette veillance est donc projective, et la bienveillance se rapproche en cela du bonheur qui «n'est jamais goûté au présent mais seulement dans l'aprèscoup » (Clavier, Rey, 2005).

31 Enfin, dans ce recueil d'expression sur les pratiques, des invariants sont apparus : les enseignants ont à chaque fois parlé de l'accueil réservé aux élèves, de l'importance de l'écoute, du dialogue, de la façon dont ils veillaient à les comprendre en étant attentif et en les observant, à valoriser leur travail. Ils ont aussi souligné l'importance de la confiance et de la reconnaissance. Ces éléments récurrents apparaissent donc comme constitutifs de la bienveillance. 


\section{Faire preuve de bienveillance : une dynamique d'ajustements} adaptations que le personnel enseignant met en œuvre dans ses démarches d'actualisation de la bienveillance auprès des élèves. Ces adaptations s'inscrivent dans une dynamique d'ajustement qui préside aux pratiques relatées. Nous avons pu identifier un ajustement en fonction du contexte des élèves accueillis, en fonction de leur âge et selon le cycle de scolarisation, mais aussi selon le contexte d'exercice. Ainsi, Fabrice explique que la bienveillance répond à une nécessité qui peut être variable selon le profil de l'élève. Trois contextes de scolarisation différents ont été mentionnés comme nécessitant une prise en compte particulière des élèves et, de ce fait, une actualisation spécifique : les collèges appartenant au Réseau d'Éducation Prioritaire dans lesquels sont scolarisés beaucoup d'enfants dont les familles appartiennent à des catégories socio-professionnelles défavorisées; les Sections d'Enseignement Général et Professionnel Spécialisé (SEGPA) qui accueillent des enfants ayant de grandes difficultés d'apprentissage; les situations des élèves allophones nouvellement arrivés en France et qui ne maîtrisent pas la langue française.

La bienveillance constitue alors un moyen d'assurer la proximité nécessaire au développement des apprentissages, en réduisant la distance entre les élèves et les familles et l'École.

Le personnel enseignant a aussi exprimé les limites inhérentes à l'actualisation de la bienveillance, notamment son caractère aléatoire, et la difficulté de son évaluation. Il a souligné la variabilité éprouvée dans sa capacité à faire preuve de bienveillance. François précise par exemple: «on peut l'être à des degrés divers, en fonction des personnes, de la temporalité, au début à la fin de l'année ». Cette variabilité des paramètres à prendre en compte montre que la seule intentionnalité de bienveillance ne donne aucune garantie de succès. De ce fait, la nécessaire bienveillance par rapport à soi a été plusieurs fois soulignée.

Enfin, l'analyse des données a permis de mettre en évidence des caractéristiques de la professionnalité qui se dégagent à partir des différentes contributions aux entrevues. La bienveillance invite à une posture réflexive empreinte d'ajustement et de souplesse, et donc à un positionnement professionnel particulier. Elle prend appui sur une posture construite sur un ensemble de qualités professionnelles qui sous-tendent les pratiques sur le terrain : le tact (Prairat, 2017) et la prudence, qui se concrétisent dans la retenue du jugement et du langage, la clairvoyance et la sagacité qui font découvrir et comprendre les choses les plus difficiles, la persévérance et la constance dans l'établissement des relations, l'hospitalité, la patience, la vigilance et la sincérité, qui sont autant de qualités construites qui viennent soutenir la bienveillance et peuvent contribuer à une plus grande félicité scolaire.

\section{Conclusion}

Partant de l'inflation de la notion de bienveillance du champ social au domaine scolaire, nous avons montré les liens entre cet appel et la quête actuelle de bien-être, d'épanouissement et de davantage de félicité à l'École. Nous avons proposé une caractérisation de la notion en nous appuyant sur l'éthique professionnelle, soulignant 
son caractère difficilement saisissable notamment dans les pratiques. Nous avons ensuite présenté les résultats de recherche qui permettent de caractériser l'opérationnalisation de la bienveillance dans les pratiques professionnelles du personnel enseignant de collège, à partir de leurs discours. Au travers des différentes formes de bienveillance et de la dynamique d'ajustement, nous pouvons constater l'exigence professionnelle nécessaire à ces pratiques.

En réponse à nos interrogations initiales, nous avons pu mettre à jour différentes manières d'opérationnaliser la bienveillance. Au-delà de nouvelles modalités relationnelles et d'une simple histoire de qualité personnelle, nous avons pu voir que la bienveillance s'inscrit dans des modalités pédagogiques et didactiques exigeantes et spécifiques qui induisent une posture d'ajustement particulière. Ses manifestations dans l'acte éducatif vont donc bien au-delà de la seule expression du visage. Elles s'incarnent en prenant appui sur les dimensions que nous avons mises à jour. En effet, la bienveillance comme art professoral et la bienveillance comme incarnation du cadre s'appuient sur la dimension intentionnelle: elles se déclinent dans une relation finalisée par la responsabilité éducative et orientée par la préoccupation du "en vue de » et du « afin de » du développement des élèves. Elles prennent aussi toutes deux appui sur la dimension interactionnelle car elles nécessitent un ajustement aux personnes et aux situations pour permettre l'effectivité de la bienveillance. Enfin, elles sont nourries par les dimensions attentionnelles et affectives, chacune à leur manière, dimensions qui facilitent la perception et conditionnent l'ajustement professionnel. Le lien entre les modalités et les dimensions permet également d'inscrire la proximité de la bienveillance avec l'éthique du care, éthique relationnelle et réflexive soucieuse du maintien et du lien et qui laisse place à la sensibilité et aux émotions.

Toutefois, notre entreprise de «saisie » de la bienveillance s'est trouvée confrontée à certaines limites, notamment du fait de tenter d'accéder à une notion ancrée dans l'intentionnalité, et donc dans l'intériorité. La dimension compréhensive et exploratoire de cette recherche rend ses conclusions encore hypothétiques et très certainement provisoires, même si ses résultats sont congruents avec ceux de Saillot (2018). Pour approfondir la compréhension de la bienveillance proposée, il serait intéressant, d'une part, de chercher à vérifier la possibilité d'extension de nos résultats par une recherche quantitative. Il serait, d'autre part, important de ne pas limiter les données à un déclaratif sur les pratiques mais de les étendre à des observations de situations de travail.

Nous estimons avoir contribué au renforcement de la caractérisation de la bienveillance, ce qui nous amène à revenir au lien entre la bienveillance, le bien-être et plus largement la quête actuelle de félicité pour l'École. Si le déploiement de ces notions n'est pas aisé à mettre en œuvre et à percevoir dans les pratiques professionnelles, cet appel à ces notions positives présage de changements forts pour le système scolaire français. Les évolutions éducatives sous-jacentes visent ainsi à la fondation d'une école davantage hospitalière et inclusive, invitant à construire une école davantage humaniste et à un changement de paradigme éducationnel. Il ne s'agit toutefois pas d'une conception statique du bonheur à l'École. Au contraire, cet appel nous ouvre à une projection dynamique dans laquelle le désir d'une École meilleure prime et donne une orientation. Le système sera long à changer, mais les changements peuvent prendre partiellement appui sur la force transformatrice de la bienveillance 
qui, pour Spaemann, est "pour l'homme bienveillant lui-même, un cadeau. Elle est l'eudaimonia, la réussite de la vie » (p.145).

\section{BIBLIOGRAPHIE}

Aubert, N. (dir.) (2004). L’individu hypermoderne. Toulouse, France : Érès.

Bergounioux, A., Loeffel, L. et Schwartz, R. (2013). Pour un enseignement laïque de la morale. Paris, France : MGEN.

Bergugnat, L. (2017). Penser le bien-être à l'école : en enjeu éthique ? In Lasplacettes, G. (dir) (2017). Prendre soin, santé et bien-être en éducation. Diversité (189) 73-77.

Cabanas, E. et Illous, E. (2018). Happycratie. Comment l'industrie du bonheur a pris le contrôle de nos vies. Paris, France : Premier Parallèle.

Clavier, P. Rey, A. (2005). Bonheur. In Rey (dir.), Dictionnaire culturel en langue française. (Vol.2, p. 991- 996). Paris, France : Le Robert.

Crahay, M. (2006). Qualitatif - quantitatif : des enjeux méthodologiques convergents? In L. Paquay, M. Crahay et J.-M. de Ketele (dir.), L'analyse qualitative en éducation (p. 33-52). Bruxelles, Belgique : De Boeck, Collection pédagogies en développement.

Debarbieux, E. (2015). Du « climat scolaire » : définitions, effets et politiques publiques. In F. Murat et C. Simonis-Sueur (2015), Climat scolaire et bien-être à l'école. Education \& Formations, $n^{\circ} 88-89$. Paris, France : MENSR.

Defrance, B. (1992). La violence à l'école. Paris, France : Syros/Alternatives.

Ehrenberg, A. (1998). La fatigue d'être soi : dépression et société. Paris, France : Odile Jacob.

Florin, A. et Bacro, F. (2017) Bien-être et qualité de vie : prendre soin des enfants. In Lasplacettes, G. (dir) (2017). Prendre soin, santé et bien-être en éducation. Diversité (189) 48-55.

Foulquié, P. et Saint-Jean, R. (1969). Dictionnaire de la langue philosophique (2éd.). Paris, France : Presses Universitaires de France.

Geoffrion, P. (2016). Le groupe de discussion. In B. Gauthier (dir.), Recherche sociale. De la problématique à la collecte de données (p. 401 à 426). Montréal, Canada : Presses de l'Université du Québec.

Gueguen, C. (2014). Pour une enfance heureuse : repenser l'éducation à la lumière des dernières découvertes sur le cerveau. Paris, France : Robert Laffont.

Jellab, A. (2018). La bienveillance, un nouveau care en éducation. In A. Jellab et C. Marsollier (dir). (2018). Bienveillance et bien-être à l'école, plaidoyer pour une école humaine et exigeante. (p. 19-56). Paris, France : Editions Berger-Levrault.

Marquis, N. (2014). Du bien-être au marché du malaise : la société du développement personnel. Paris, France : Presses Universitaires de France. 
Marsollier, C. (2018). De la nécessité d'accorder toute sa valeur éthique à la bienveillance active. In A. Jellab et C. Marsollier (dir). (2018). Bienveillance et bien-être à l'école, plaidoyer pour une école humaine et exigeante. (p. 57-79). Paris, France : Editions Berger-Levrault.

Massé, C. (1986). L'apprentissage de la bienveillance en éducation morale. Mémoire de maîtrise non publié, Université Laval, Canada.

MEN (2013). Référentiel des compétences professionnelles des métiers du professorat et de l'éducation. (MENE1315928A - arrêté du 1-7-2013 - J.O. du 18-7-2013). En Ligne, http:// www.education.gouv.fr/pid285/bulletin_officiel.html?cid_bo=73066

Merle, P. (2005). L'élève humilié : l'école, un espace de non-droit ? Paris, France : Presses Universitaires de France.

Miles, M. B. et Huberman, A. M. (2003). Analyse des données qualitatives. Bruxelles, Belgique : De Boeck Supérieur.

Mons, N. (dir.) (2016). Inégalités sociales et migratoires. Comment l'école amplifie-t-elle les inégalités? Rapport du CNESCO. Paris, France : Ministère de l'Éducation nationale de l'Enseignement Supérieur et de la Recherche.

Moulias, R., Moulias S. et Busby F., (2010). La « bientraitance » : qu'est-ce que c'est ? Gérontologie et société, 2(133), 10-21.

Nguyen T. (2016). La qualité de vie et le bien-être à l'école en France : quelle place dans les textes institutionnels? Paris, France: CNESCO

Noddings, N. (1984). Caring, A relational approach to Ethics and Moral Education. Berkeley, Etats-Unis : University of California Press.

Paillé, P. et Mucchielli, A. (2012). L'analyse qualitative en sciences humaines et sociales. Paris, France : Armand Colin.

Paillé, P. (1991). Procédures systématiques pour l'élaboration d'un guide d'entrevue semi-directive : un modèle et une illustration. Communication au Congrès de l'Association canadienne-française pour l'avancement des sciences (ACFAS). Sherbrooke, Québec, Canada : Université de Sherbrooke.

Poché, F. (2016). De l'hyper-vulnérabilité. Diagnostic du présent et clarification conceptuelle. Revue des sciences religieuses, 90(1), 51-61.

Prairat, E. (2017). Éduquer avec tact. Paris, France : ESF - Sciences humaines.

Prairat, E. (2014). L'éthique de l'enseignement, enjeux personnels, professionnels et institutionnels. Nancy, France : Presses universitaires de Lorraine.

Rascle, N., Bergugnat, L., Florin, A. et Guimard, Ph. (2016). Qualité de vie des enseignants en relation avec celle des élèves : revue de question, recommandations. Paris, France : Cnesco.

Réto, G. (2018). La bienveillance dans le champ scolaire, Caractérisation des pratiques et actualisation selon des membres du personnel enseignant de collège, des chefs d'établissement et des experts du monde de l'éducation. Thèse de doctorat en cotutelle. Université de Sherbrooke (Canada), Université Catholique de l'Ouest (Angers, France).

Réto, G. (2017). La bienveillance à l'Ecole : vers un changement de paradigme ? Recherches \& éducations, 18. En ligne http://journals.openedition.org/rechercheseducations/4389

Réto, G. (2016). Le caring, une voie pour reconfigurer l'École française au moment de sa refondation ? Éducation et socialisation. En ligne : http://edso.revues.org/1504 
Saillot, E. (2018) (à paraître). Conforter une école bienveillante et exigeante : représentations, préoccupations et pratiques déclarées. Questions Vives, 29.

Savoie-Zajc, L. (2016). L'entrevue semi-dirigée, In B. Gauthier et I. Bourgeois (dir.), Recherche sociale. De la problématique à la collecte de données (p. 337-362). Québec, Canada : Presses de l'Université de Québec.

Spaemann, R. (1997). Bonheur et Bienveillance, essai sur l'éthique (trad. par S. Robillard). Paris, France : Presses Universitaires de France.

Taylor, C. (1998). Les sources du moi : la formation de l'identité moderne (trad. par C. Melançon). Paris, France : Seuil.

\section{NOTES}

1. Cet article prend appui sur un travail de thèse consacré à la bienveillance dans le champ scolaire (Réto, 2018).

2. Contrairement au primaire, les élèves ont un ensemble de professeurs avec lesquels il s'agit de tisser des relations spécifiques. De plus, s'ils font partie d'une même communauté éducative, les enseignants ont développé une gamme de pratiques dont la variété est intéressante à développer. 3. Conformément aux attendus posés par le Comité d'Ethique de la Recherche de l'Université de Sherbrooke, nous avons adressé en amont un formulaire d'information et de consentement à toutes les personnes que nous souhaitions recruter (présentation de la recherche, de ses objectifs et des enjeux de la divulgation de l'information recueillie) et chaque participante ou participant a signé et conservé un exemplaire cosigné.

4. Les prénoms sont des prénoms d'emprunt.

5. Axe 1: définition de la bienveillance - axe 2: actualisation de la bienveillance dans les pratiques de classe - Axe $3:$ lien entre bienveillance et projet institutionnel - Axe $4:$ atouts et limites de la bienveillance dans le champ scolaire. Pour plus de détails, nous renvoyons à la thèse (Réto, 2018) à laquelle les guides sont annexés.

6. Il s'est agi d'une analyse thématique prenant appui sur Paillé et Mucchielli (2012). Un premier volet a constitué en un examen puis en une réduction phénoménologique des données, conduisant à une thématisation progressive en continue qui a été structurée et hiérarchisée, permettant la réalisation d'un arbre thématique avec grappes de thèmes saillants. Un deuxième volet a été mené à la suite du premier et a pris appui sur le logiciel QDA Miner, logiciel d'analyse qualitative développé par la société Provalis. Ce second volet a permis d'affermir l'analyse et de confirmer ou d'infirmer la saillance thématique. Si les deux volets ont été menés successivement, il s'est bien agi d'un processus analytique par combinaison et articulation.

\section{RÉSUMÉS}

Dans le sillage du bonheur, la bienveillance soulève aujourd'hui un engouement dont l'évidence mérite un éclairage porté par un souci d'objectivation. L'article met en relief l'émergence de cette notion dans la société puis dans le cadre de l'École. Il montre l'appel fait à la bienveillance qui existe au sein du système scolaire, notamment dans le projet pour la refondation de l'École 
française, et interroge le sens de cet appel. Il conduit au constat du manque de conceptualisation de la notion, ce qui rend complexe son opérationnalisation tant au niveau de l'institution que sur le plan des pratiques au sein des établissements scolaires. Une clarification de la notion de bienveillance est alors proposée en l'inscrivant dans le champ de la philosophie, et plus précisément de l'éthique. Afin de caractériser les pratiques relevant de la bienveillance à l'École, l'article prend alors appui sur une recherche exploratoire menée auprès de membres du personnel enseignant travaillant dans des collèges. S'appuyant sur des entrevues, elle permet de caractériser les pratiques de bienveillance du personnel enseignant. Du constat de la mollesse de la notion à sa caractérisation, l'article conduit à mettre en évidence les changements importants reliés à cet appel à la bienveillance et aux notions qui lui sont reliées dans les pratiques à l'École française.

In the track of happiness, these days benevolence causes such a profound stir of passions that it demands a new focus founded in objectification. This paper highlights the emergence of the notion firstly within society, then within the field of Education and lastly within the framework of the French school. It demonstrates the need for benevolence in existence within the school system, particularly within the project entitled "Refondation de l'École française" (new foundations for the school system in France). It queries the meaning of this direction. The lack of conceptualization at stake is deplored which in turn affects the management at institutional level and in the practices at school level. The essence of the meaning of the term benevolence is therefore suggested when one situates it within the realm of the philosophical and of Ethics in particular. To characterize practices, the research was conducted with staff members. Embedded within interviews, the practices of benevolence are being characterized. From the acknowledgement of the definition of benevolence as a soft notion to its characterization, the paper points to the important changes connected to the claim of benevolence and close notions within the practices of the French school system.

\section{INDEX}

Mots-clés : bienveillance, relation éducative, pratiques d'enseignement, éthique professionnelle

Keywords : benevolence, educational relationships, educational practices, professional ethics

\section{AUTEUR}

\section{GWÉNOLA RÉTO}

Université Catholique de l'Ouest, PESSOA 